\title{
Addition of Thiols to Phenylselenoalkynes using KF/Alumina under Solvent-Free Conditions
}

\author{
Renata G. Lara, Elton L. Borges, Eder J. Lenardão, Diego Alves, \\ Raquel G. Jacob and Gelson Perin* \\ Instituto de Química e Geociências, LASOL, Universidade Federal de Pelotas, \\ CP 354, 96010-900 Pelotas-RS, Brazil
}

\begin{abstract}
Um método simples e eficiente foi desenvolvido para a hidrotiolação de fenilselenoalquinos utilizando $\mathrm{KF} / \mathrm{Al}_{2} \mathrm{O}_{3}$ em meio livre de solvente. $\mathrm{O}$ método é geral e permite a preparação seletiva de (Z)-1-fenilseleno-2-organotio-1-alquenos a partir de tióis aromáticos e alifáticos em rendimentos de razoáveis a bons. A presença do grupamento organosselênio direcionou a regioquímica da adição do tiol. O sistema catalítico pode ser reutilizado mais quatro vezes sem tratamento prévio.

We present herein the results of a simple and efficient protocol for the hydrothiolation of phenylselenoalkynes promoted by $\mathrm{KF} / \mathrm{Al}_{2} \mathrm{O}_{3}$ using solvent-free conditions. This improved method furnishes selectively the corresponding $(Z)$-1-phenylseleno-2-organylthio-1-alkenes in reasonable to good yields starting from selenoalkynes and aliphatic or aromatic thiols. The presence of the phenylselenium group in the alkyne directed the regiochemistry of the thiol addition. The catalytic system can be reused up to 4 times without previous treatment.
\end{abstract}

Keywords: selenium, green chemistry, solvent-free reaction, hydrothiolation, vinyl selenides

\section{Introduction}

The development of new preparative methods for vinyl chalcogenides is still being of interest in organic synthesis and in materials science. ${ }^{1}$ Among the vinyl chalcogenides the densely functionalized 1,2-bis-chalcogenide alkenes are of special interest, because they can be used as a versatile precursor to enediynes and other functionalized olefins. ${ }^{2}$ The methods for preparation of vicinal bischalcogenide alkenes involve the addition of two identical organochalcogenium groups in alkynes. This reaction can be catalyzed by transition metal complexes $\left[\mathrm{Pd}\left(\mathrm{PPh}_{3}\right)_{4}\right]$ and $\left[\mathrm{Pt}\left(\mathrm{PR}_{3}\right)_{4}\right],{ }^{3}$ gallium trichloride, ${ }^{4}$ or under photochemical, ${ }^{5}$ thermal ${ }^{6}$ or basic conditions. ${ }^{7}$ Because the difference in reactivity of the organoselenium and organosulfur groups attached at the double bond, the preparation of mixed ( $\mathrm{S}$, $\mathrm{Se}$ ) bis-chalcogenide alkenes was also developed. ${ }^{3,5}$

Recently, the in situ addition of chalcogenides to propargylic alcohols (alkynyl-lithium species) to afford bis-chalcogenide alkenes ( $\mathrm{S}$ and $\mathrm{Se}$ ) in very good yields was described. ${ }^{8}$ The authors observed that the presence of the acidic hydrogen from hydroxyl group is essential

\footnotetext{
*e-mail: gelson_perin@ufpel.edu.br
}

for the selectivity control of the addition. An alternative method to obtain mixed vinyl bis-chalcogenides is the hydrochalcogenation of chalcogenoalkynes. ${ }^{9,10}$ In this way, sodium phenylseleno-(triethoxy)borate, generated in situ in the presence of refluxing ethanol, was used for the hydroselenation of phenylthioalkynes to prepare (Z)-1-phenylseleno-2-phenylthio-1-organylethenes. ${ }^{9}$ The authors observed that the phenylthio group acts as a directing and activating group for the nucleophilic addition of the selenolate species. Recently, the solvent-free hydrothiolation of thioacetylenes catalyzed by $\mathrm{PhSeBr}$ was used for the selective formation of (Z)-1,2-bisorganylthio alkenes in good yields. ${ }^{10}$ The authors studied also the hydrothiolation of methylselenoalkyne (only one example) and they observed the exclusive formation of the respective (Z)-1-phenylthio-2-methylseleno-1organylalkene..$^{10}$

On other hand, the use of potassium fluoride supported on alumina $\left(\mathrm{KF} / \mathrm{Al}_{2} \mathrm{O}_{3}\right)$ as a green catalytic system for a number of transformations has been increased. ${ }^{11}$ By using $\mathrm{KF} / \mathrm{Al}_{2} \mathrm{O}_{3}$, the products can be easily isolated by filtration and the generation of large amounts of salts at the end of the synthesis, as well as the use of stoichiometric strong bases, can be avoided. 
Our major research goal has been the development of new and cleaner protocols for the preparation and synthetic applications of organochalcogenium compounds. ${ }^{12}$ More recently, we have described several efficient approaches using $\mathrm{KF} / \mathrm{Al}_{2} \mathrm{O}_{3} \cdot{ }^{13}$ As a continuation of our studies, we report herein the results of the hydrothiolation of phenylselenoalkynes $\mathbf{1}$ using $\mathrm{KF} / \mathrm{Al}_{2} \mathrm{O}_{3}$ for the selective synthesis of (Z)-1-organylthio-2-phenylseleno1-organylalkenes 3 (Scheme 1).

\section{Results and Discussion}

Initially, we chose 1-phenylseleno-2-phenylethyne 1a and benzenethiol 2a as standard starting materials to establish the best reaction conditions (Table 1). We examined the temperature, amount of $\mathrm{KF} / \mathrm{Al}_{2} \mathrm{O}_{3}(50 \%)^{14}$ and the use of $\mathrm{N}_{2}$ atmosphere. It was found that stirring a mixture of $1 \mathbf{a}(1 \mathrm{mmol})$ and $\mathbf{2 a}(1 \mathrm{mmol})$ in presence of $0.08 \mathrm{~g}$ of $\mathrm{KF} / \mathrm{Al}_{2} \mathrm{O}_{3}(50 \%)$ at room temperature, the products of hydrothiolation 3a were obtained in a overall yield of $30 \%$ after $3.5 \mathrm{~h}$, together with great amount of diphenyl disulfide (Table 1, entry 1). When the same protocol was performed at gently heating $\left(60{ }^{\circ} \mathrm{C}\right)$ and under $\mathrm{N}_{2}$ atmosphere, the yield increased to $58 \%$ yield (Table 1, entry 3 ). On the other hand, using 1.2, 1.5 and 2 equiv. of $\mathbf{2 a}$, the yield increased to 68,78 and $90 \%$, respectively (Table 1, entries 5, 6 and 7). When an excess of phenylselenoalkyne 1a was used, unsatisfactory yields were obtained (Table 1 , entries 2 and 4), while using $0.10 \mathrm{~g}$ of the catalyst (entry 8 ) or prolonged reaction time (entry 9 ) also did not increase significantly the vinyl sulfides yields. It was also observed that the catalytic system can be re-used for several cycles, just by washing it with ethyl acetate and drying under vacuum. The product 3a was obtained in 90, 87, 78, 67 and $60 \%$ yields after successive cycles.

Using the optimized conditions, the protocol was extended to other thiols (Table 2, entries 2-4) and 1-phenylseleno heptyne 1b (Table 2, entries 5-8). ${ }^{15}$ Starting from disubstituted selenoalkynes $\mathbf{1 a}$ and $\mathbf{1} \mathbf{b}$, the hydrothiolation reaction afforded trisubstituted olefins, which can be present as four different isomers. However, we observed here that, except for the reaction between $\mathbf{1 b}$ and 2a, which formed also the gem-1,2-bis-chalcogenide (trace amount detected by GC), for all the studied examples, it
Table 1. Optimization of the synthesis of $\mathbf{3 a}$

\begin{tabular}{lccccc}
\hline entry & $\begin{array}{c}\mathbf{1 a} \\
\text { (equiv.) }\end{array}$ & $\begin{array}{c}\mathbf{2 a} \\
\text { (equiv.) }\end{array}$ & Temp. & $\begin{array}{c}\text { time } \\
(\mathrm{h})\end{array}$ & $\begin{array}{c}\text { Yield } \\
(\%)^{\mathrm{a}}\end{array}$ \\
\hline 1 & 1 & 1 & r.t. & 3.5 & 30 \\
$2^{\mathrm{b}}$ & 2 & 1 & r.t. & 3.5 & 38 \\
$3^{\mathrm{b}}$ & 1 & 1 & $60{ }^{\circ} \mathrm{C}$ & 3.5 & 58 \\
$4^{\mathrm{b}}$ & 2 & 1 & $60{ }^{\circ} \mathrm{C}$ & 3.0 & 65 \\
$5^{\mathrm{b}}$ & 1 & 1.2 & $60^{\circ} \mathrm{C}$ & 3.0 & 68 \\
$6^{\mathrm{b}}$ & 1 & 1.5 & $60^{\circ} \mathrm{C}$ & 3.0 & 78 \\
$7^{\mathrm{b}}$ & 1 & 2 & $60{ }^{\circ} \mathrm{C}$ & 3.0 & 90 \\
$8^{\mathrm{c}}$ & 1 & 2 & $60{ }^{\circ} \mathrm{C}$ & 3.0 & 91 \\
$9^{\mathrm{b}}$ & 1 & 2 & $60{ }^{\circ} \mathrm{C}$ & 24.0 & 90 \\
\hline
\end{tabular}

${ }^{\mathrm{a}}$ Obtained as a mixture of $(Z)-\mathbf{3 a}$ and $(E)-\mathbf{3 a}$ (ratio $\left.=70: 30\right)$. ${ }^{\mathrm{b}}$ Reactions accomplished under of $\mathrm{N}_{2}$ atmosphere. ${ }^{\circ}$ Reaction using $0.10 \mathrm{~g}$ of $\mathrm{KF} / \mathrm{Al}_{2} \mathrm{O}_{3}$.

was obtained exclusively the 1-phenylseleno-2-phenylthio1-organylethenes 3 (Table 2). This observation showed that the phenylselenium moiety acts as a directing and activating group for the nucleophilic addition of the thiolate anion. This influence in the regiochemistry of the reaction is similar to that observed for the hydrochalcogenation of thioacetylenes. ${ }^{9}$ Regarding the stereochemistry of product 3, the (Z)-isomer was obtained preferentially, with a $Z: E$ ratio around of 70:30 in all the studied examples. The best yields were obtained using the benzenethiol 2a. Thus, for example, from the reaction of phenylselenoalkyne derived from phenylacetylene, $\mathbf{1 a}$ or 1-heptyne, $\mathbf{1 b}$ with benzenethiol $\mathbf{2 a}$, the respective products $\mathbf{3 a}$ and $\mathbf{3 e}$ were obtained in $90 \%$ yield (Table 2, entries 1 and 5). When benzylic (2c and 2e) and aliphatic thiols (2d) were used, the product 3 were obtained in $49-60 \%$ yields (Table 2, entries 3-4 and 7-8).

The low yield observed for the aliphatic thiols $2 \mathbf{c}-\mathbf{e}$ compared with benzenethiol 2a can be attributed to a competition between the hydrothiolation reaction and the thiol oxidation to afford the respective disulfides. For all the studied examples, a variable amount of disulfide was isolated. This is in agreement with a recently report of our group describing the clean oxidation of thiols to disulfide in the presence of $\mathrm{KF} / \mathrm{Al}_{2} \mathrm{O}_{3} \cdot{ }^{13}$ Thus, the unreacted excess of thiol employed was easily recovered at the end of the reaction as the corresponding disulfide. A plausible mechanism for the formation of $\mathbf{3 a}-\mathbf{h}$ is outlined in

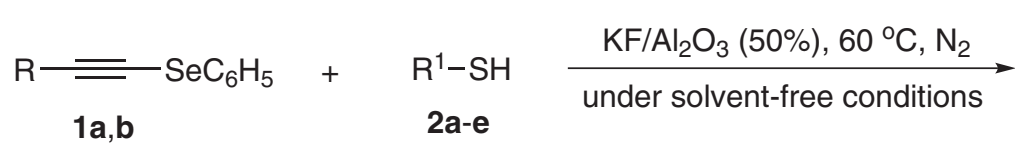

$\mathrm{R}=\mathrm{C}_{6} \mathrm{H}_{5}, \mathrm{C}_{5} \mathrm{H}_{11}$

$\mathrm{R}^{1}=\mathrm{C}_{6} \mathrm{H}_{5}, 4-\mathrm{ClC}_{6} \mathrm{H}_{4}, 4-\mathrm{ClC}_{6} \mathrm{H}_{4} \mathrm{CH}_{2}, \mathrm{C}_{6} \mathrm{H}_{5} \mathrm{CH}_{2}, \mathrm{C}_{12} \mathrm{H}_{25}$<smiles>[R15]C([R])=C[SeH]</smiles>

(Z)-3a-h

(E)-3a-h 
Table 2. Hydrothiolation of phenylselenoalkynes using $\mathrm{KF} / \mathrm{Al}_{2} \mathrm{O}_{3}$ under solvent-free condition

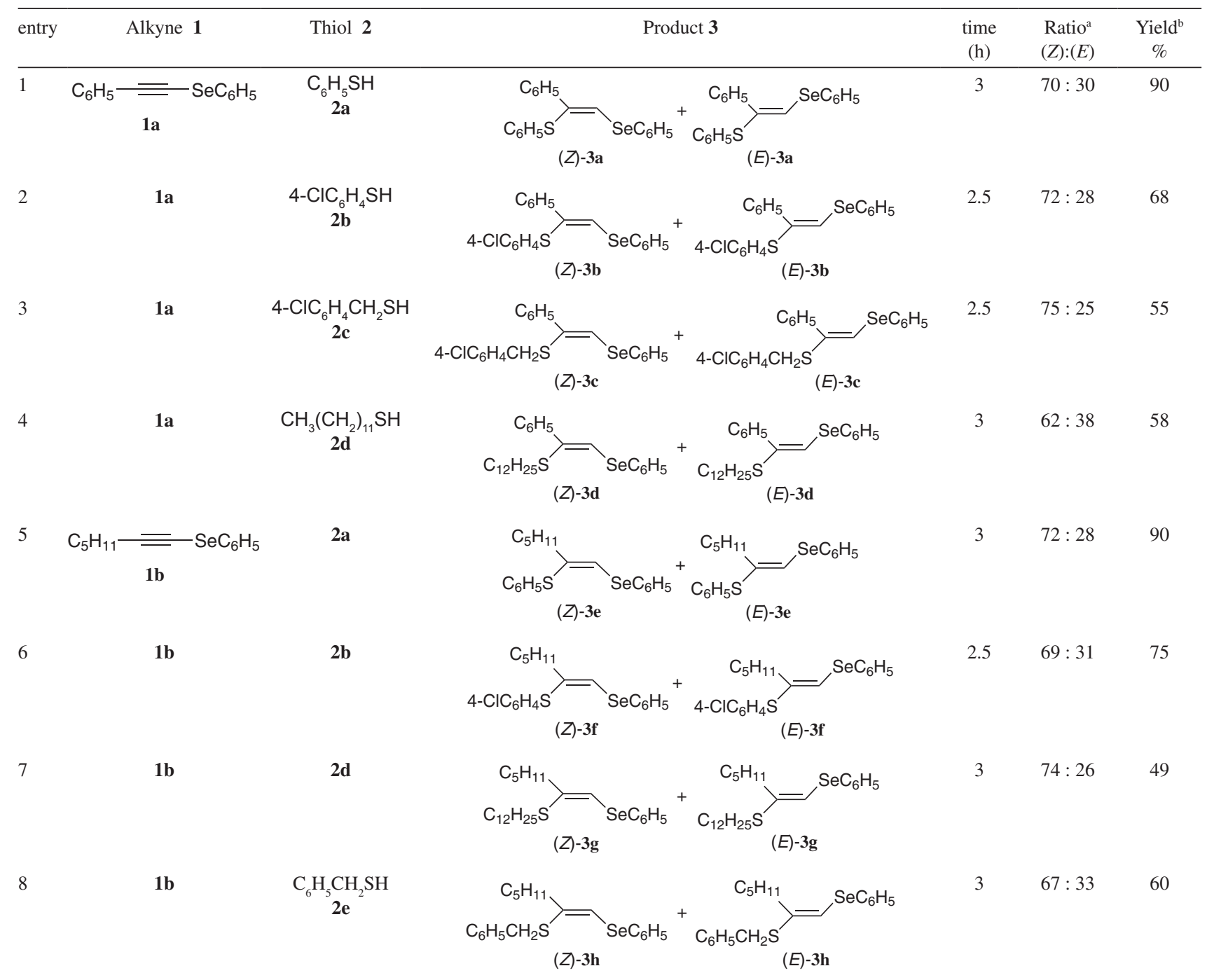

a Determined by ${ }^{1} \mathrm{H}$ NMR of the crude reaction mixture and confirmed after isolation of pure products. ${ }^{\text {b}}$ Yields of pure products isolated by column chromatography (hexanes/AcOEt).

Scheme 2. The reaction was initiated by addition of the thiolate anion to give the intermediate $\mathbf{4 a}$ and $\mathbf{4 b}$ which by abstraction of proton to give the desired products $\mathbf{3}$.

In conclusion, several (Z)-1-organylthio-2-phenylseleno1-organylalkenes could be selectively prepared starting from thiol and selenoalkynes under solvent-free conditions using $\mathrm{KF} / \mathrm{Al}_{2} \mathrm{O}_{3}$. This regio- and stereocontrolled method is general and can be used for aromatic and aliphatic thiols with reasonable to good yields. This green protocol consists in low consumption of solvent in the overall process, short

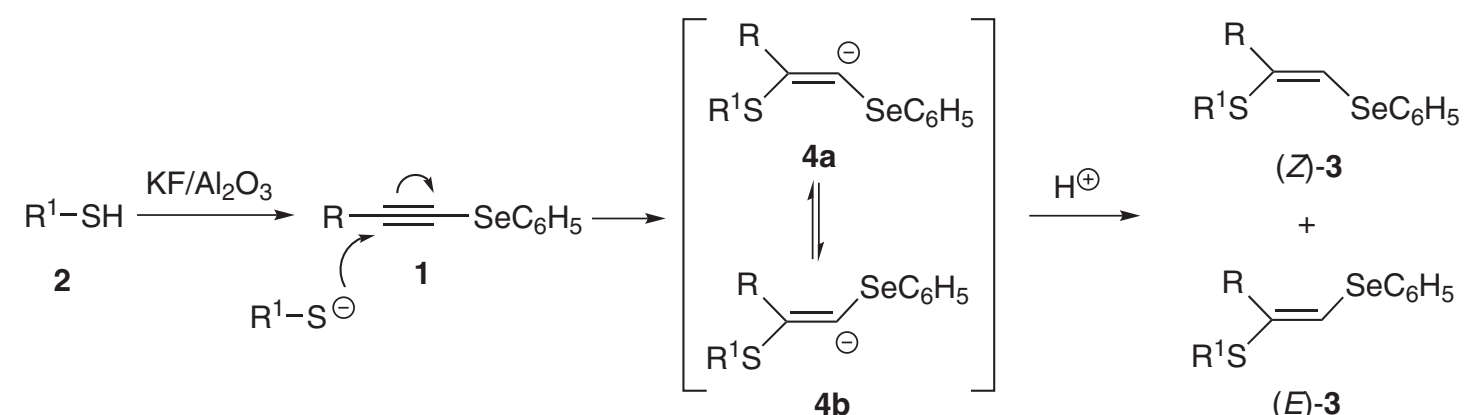

Scheme 2. 
reaction time, mild reaction conditions and simplicity, with non-aqueous work-up. The catalytic system was re-used up to 4 times with a slight declining in yields.

\section{Acknowledgments}

This project was funded by CNPq, FAPERGS/ PRONEX 10/0005-1, FINEP, and CAPES. Prof. Lopes, N. P. from FCFRP-USP, is thanked for the HRMS analyses.

\section{References}

1. For the preparation and synthetic utility of vinyl chalcogenides see, for example: Comasseto, J. V.; Ling, L. W.; Petragnani, N.; Stefani, H. A.; Synthesis 1997, 373; Zeni, G.; Lüdtke, D. S.; Panatieri, R. B.; Braga, A. L.; Chem. Rev. 2006, 106, 1032; Perin, G.; Lenardão, E. J.; Jacob, R. G.; Panatieri, R. B.; Chem. Rev. 2009, 109, 1277; Zyk, N. V.; Beloglazkina, E. K.; Belova, M. A.; Dubinina, N. S.; Russ. Chem. Rev. 2003, 72, 769; Kondo, T.; Mitsudo, T.; Chem. Rev. 2000, 100, 3205; Gavrilova, G. M.; Amosova, S. V.; Heteroatom. Chem. 2006, 17, 491; Beletskaya, I. P.; Ananikov, V. P.; Eur. J. Org. Chem. 2007, 3431.

2. Zeni, G.; Braga, A. L.; Stefani, H. A.; Acc. Chem. Res. 2003, 36, 731; Alves, D.; Schumacher, R. F.; Brandão, R.; Nogueira, C. W.; Zeni, G.; Synlett 2006, 1035; Zeni, G.; Perin, G.; Cella, R.; Jacob, R. G.; Braga, A. L.; Silveira, C. C.; Stefani. H. A.; Synlett 2002, 975; Zeni, G.; Nogueira, C. W.; Pena, J. M.; Pilissão, C.; Menezes, P. H.; Braga, A. L.; Rocha, J. B. T.; Synlett 2003, 579; Alves, D.; Zeni, G.; Nogueira, C. W.; Tetrahedron Lett. 2005, 46, 8761 .

3. Ananikov, V. P.; Beletskaya, I. P.; Aleksandrov, G. G.; Eremenko, I. L.; Organometallics 2003, 22, 1414.

4. Usugi, S.; Yorimitsu, H.; Shinokubo, H.; Oshima, K.; Org. Lett. 2004, 6, 601.

5. Ogawa, A.; Tsuboi, Y.; Obayashi, R.; Yokoyama, K.; Ryu, I.; Sonoda, N.; J. Org. Chem. 1994, 59, 1600; Ogawa, A.; Yokoyama, K.; Obayashi, R.; Han, L.-B.; Kambe, N.; Sonoda, N.; Tetrahedron 1993, 49, 1177; Back, T. G.; Krishna, M. V.; J. Org. Chem. 1988, 53, 2533; Ogawa, A.; Yokoyama, H.; Yokoyama, Masawaki, T.; Kambe, N.; Sonoda N.; J. Org. Chem. 1991, 56, 5721; Ogawa, A.; Ogawa, I.; Sonoda N.; J. Org. Chem. 2000, 65, 7682; Ogawa, A.; Obayashi, R.; Ine, H.; Tsuboi, Y.; Sonoda, N.; Hirao, T.; J. Org. Chem. 1998, 63, 881.

6. Potapov, V. A.; Amosova, S. V.; Beletskaya, I. P.; Starkova, A. A.; Hevesi, L.; Phosphorus, Sulfur, Silicon Relat. Elem. 1998, 136, 591; Kuniyasu, H.; Ogawa, A.; Miyazaki, S.; Ryu, I.; Kambe, N.; Sonoda, N.; J. Am. Chem. Soc. 1991, 113, 9796; Potapov, V. A.; Amosova, S. V.; Doron'kina, I. V.; Starkova, A. A.; Hevesi, L.; Sulfur Lett. 2002, 25, 101.

7. Moro, A. V.; Nogueira, C. W.; Barbosa, N. B. V.; Menezes, P. H.; Rocha, J. B. T.; Zeni, G.; J. Org. Chem. 2005, 70, 5257.
8. Schneider, C. C.; Godoi, B.; Prigol, M.; Nogueira, C. W.; Zeni, G.; Organometallics 2007, 26, 4252.

9. Dabdoub, M. J.; Dabdoub, V. B.; Pereira, M. A.; Tetrahedron Lett. 2001, 42, 1595.

10. Manarin, F.; Roehrs, J. A.; Prigol, M.; Alves, D.; Nogueira, C. W.; Zeni, G.; Tetrahedron Lett. 2007, 48, 4805.

11. Basu, B.; Das, P.; Das, S.; Curr. Org. Chem. 2008, 12, 141; Blass, B. E.; Tetrahedron 2002, 58, 9301.

12. Perin, G.; Jacob, R. G.; Dutra, L. G.; Azambuja, F.; Santos, G. F. F.; Lenardão, E. J.; Tetrahedron Lett. 2006, 47, 935; Lenardão, E. J.; Dutra, L. G.; Saraiva, M. T.; Jacob, R. G.; Perin, G.; Tetrahedron Lett. 2007, 48, 8011; Perin, G.; Jacob, R. G.; Azambuja, F.; Botteselle, G. V.; Siqueira, G. M.; Freitag, R. A.; Lenardão, E. J.; Tetrahedron Lett. 2005, 46, 1679; Lenardão, E. J.; Silva, M. S.; Mendes, S. R.; Azambuja, F.; Jacob, R. G.; Santos, P. C. S.; Perin, G.; J. Braz. Chem. Soc. 2007, 18, 943.

13. Lenardão, E. J.; Ferreira, P. C.; Jacob, R. G.; Perin, G.; Leite, F. P. L.; Tetrahedron Lett. 2007, 48, 6763; Lenardão, E. J.; Lara, R. G.; Silva, M. S.; Jacob, R. G.; Perin, G.; Tetrahedron Lett. 2007, 48, 7668; Perin, G.; Jacob, R. G.; Botteselle, G. V.; Kublik, E. L.; Lenardão, E. J.; Cella, R.; Santos, P. C. S.; J. Braz. Chem. Soc. 2005, 16, 857; Silva, M. S.; Lara, R. G.; Marczewski, J. M.; Jacob, R. G.; Lenardão, E. J.; Perin, G.; Tetrahedron Lett. 2008, 49, 1927; Lenardão, E. J.; Trecha, D. O.; Ferreira, P. C.; Jacob, R. G.; Perin, G.; J. Braz. Chem. Soc. 2009, 20, 93.

14. Preparation of alumina supported potassium fluoride: ${ }^{16}$ To a $100 \mathrm{~mL}$ beaker was added alumina $\left(4.0 \mathrm{~g}\right.$ of $\mathrm{Al}_{2} \mathrm{O}_{3} 90,0.063-$ $0.200 \mathrm{~mm}$, Merck), $\mathrm{KF} \cdot 2 \mathrm{H}_{2} \mathrm{O}(6.0 \mathrm{~g})$ and water $(10 \mathrm{~mL})$. The suspension was stirred for $1 \mathrm{~h}$ at $65^{\circ} \mathrm{C}$, dried at $80^{\circ} \mathrm{C}$ for $1 \mathrm{~h}$ and for an additional $4 \mathrm{~h}$ at $300{ }^{\circ} \mathrm{C}$ in an oven and then cooled in a desiccator. The content of KF is about $50 \%(\mathrm{~m} / \mathrm{m})$.

15. General procedure for the synthesis of 1,2-bis-chalcogenoalkenes 3: To a mixture of phenylselenoalkyne 1 (1 mmol) and the appropriate thiol $2(2 \mathrm{mmol})$ under $\mathrm{N}_{2}$ atmosphere, $\mathrm{KF} / \mathrm{Al}_{2} \mathrm{O}_{3}{ }^{14}(0.08 \mathrm{~g})$ was added at room temperature. Then, the temperature was slowly raised to $60{ }^{\circ} \mathrm{C}$. The reaction progress was followed by TLC. After consumption the starting materials (see Table 2), the crude product was filtered off the solid supported catalyst by washing with ethyl acetate $(10 \mathrm{~mL})$. The solvent was evaporated under reduced pressure and the residue was purified by column chromatography over silica gel eluting with hexane/ethyl acetate (98:2). (Z)- and (E)-3a: ${ }^{1} \mathrm{H}$ NMR (300 MHz, $\left.\mathrm{CDCl}_{3}\right) \delta \mathrm{Z}$ isomer: 7.13-764 (m, 15H), 7.60 (s, $1 \mathrm{H})$; $E$ isomer: 7.08-764 (m, 15H), $7.07(\mathrm{~s}, 1 \mathrm{H}) ;{ }^{13} \mathrm{C}$ NMR (75 MHz, $\left.\mathrm{CDCl}_{3}\right) \delta E+Z$ isomers: $140.5,138.6,137.2$, 136.1, 135.6, 134.3, 133.6, 133.2, 133.1, 131.0, 130.9, 130.8, 130.4, 129.4, 129.2, 129.1, 129.0, 128.9, 128.8, 128.7, 128.3, 128.2, 128.1, 128.0, 127.8, 127.7, 127.6, 127.5, 127.4, 127.2, 126.7, 126.6, 125.9, 125.8; MS m/z (rel. int., \%) $Z$ isomer: 368 (M+, 36.4), 259 (11.2), 209 (10.8), 178 (100.0); $E$ isomer: 368 (M+, 29.5), 259 (8.3), 209 (13.5), 178 (100.0); HRMS (ESI): 
$m / z$ calcd for $\mathrm{C}_{20} \mathrm{H}_{16} \mathrm{SSe}[\mathrm{M}+\mathrm{H}]^{+}: 369.0216$; found: 369.0222 . (Z)- and (E)-3b: ${ }^{1} \mathrm{H}$ NMR (300 MHz, $\left.\mathrm{CDCl}_{3}\right) \delta Z$ isomer: 7.11$758(\mathrm{~m}, 14 \mathrm{H}), 7.51(\mathrm{~s}, 1 \mathrm{H}) ; E$ isomer: 7.10-7.64 (m, 14H), 7.14 (s, $1 \mathrm{H}) ;{ }^{13} \mathrm{C} \mathrm{NMR}\left(75 \mathrm{MHz}, \mathrm{CDCl}_{3}\right) \delta E+Z$ isomers: 126.6 , 126.7, 127.1, 127.5, 128.0, 128.1, 128.3, 128.5, 128.5, 128.6, 128.8, 128.8, 128.9, 129.0, 129.3, 129.4, 129.4, 129.5, 129.6; MS m/z (rel. int., \%) $Z$ isomer: $402\left(\mathrm{M}^{+}, 17.4\right), 259$ (31.4), 179 (100.0), 157 (30.0), 77 (54.5); $E$ isomer: $402\left(\mathrm{M}^{+}, 20.8\right), 210$ (100.0), 77 (32.3); HRMS (ESI): $\mathrm{m} / z$ calcd for $\mathrm{C}_{20} \mathrm{H}_{15} \mathrm{ClSSe}[\mathrm{M}$ $+\mathrm{K}-\mathrm{Cl}]^{+}$: 405.9697; found: 405.2175. (Z)- and (E)-3c: ${ }^{1} \mathrm{H}$ NMR (300 MHz, $\left.\mathrm{CDCl}_{3}\right) \delta Z$ isomer: 7.12-7.63 (m, 14H), $7.60(\mathrm{~s}$, $1 \mathrm{H}) ; 3.55$ (s, 2H); $E$ isomer: 7.00-7.63 (m, 14H), $7.03(\mathrm{~s}, 1 \mathrm{H})$; $3.92(\mathrm{~s}, 2 \mathrm{H}) ;{ }^{13} \mathrm{C} \mathrm{NMR}\left(75 \mathrm{MHz}, \mathrm{CDCl}_{3}\right) \delta \mathrm{Z}+E$ isomers: 42.4, 126.6, 127.2, 127.4, 127.8, 128.2, 128.6, 129.1, 129.3, 130.6, 130.8, 133.1, 133.3, 135.7, 136.1. MS $m / z$ (rel. int., \%) $Z$ isomer: $416\left(\mathrm{M}^{+}, 15.2\right), 259$ (39.2), 178 (100.0), 157 (42.9); $E$ isomer: $416\left(\mathrm{M}^{+}, 17.4\right), 259$ (35.2), 178 (100.0), 157 (32.1); HRMS (ESI): $\mathrm{m} / \mathrm{z}$ calcd for $\mathrm{C}_{21} \mathrm{H}_{17} \mathrm{ClSSe}[\mathrm{M}+\mathrm{Na}-\mathrm{Cl}]^{+}: 405.0147$; found: 405.2218. (Z)- and (E)-3d: ${ }^{1} \mathrm{H} \mathrm{NMR}\left(300 \mathrm{MHz}, \mathrm{CDCl}_{3}\right)$ $\delta Z$ isomer: 7.14-7.65 (m, 10H); $7.60(\mathrm{~s}, 1 \mathrm{H}) ; 1.53-1.55(\mathrm{~m}$, $2 \mathrm{H})$; $1.25-1.31(\mathrm{~m}, 20 \mathrm{H}) ; 0.88$ (t, $J 7.0,3 \mathrm{H})$; $E$ isomer: 7.11$7.65(\mathrm{~m}, 10 \mathrm{H}) ; 7.06(\mathrm{~s}, 1 \mathrm{H}) ; 1.53-1.55(\mathrm{~m}, 2 \mathrm{H}) ; 1.25-1.31(\mathrm{~m}$, 20H); $0.88(\mathrm{t}, J 7.0,3 \mathrm{H}) ;{ }^{13} \mathrm{C} \mathrm{NMR}\left(75 \mathrm{MHz}, \mathrm{CDCl}_{3}\right) \delta E+Z$ isomers: 28.1, 29.08, 29.1, 29.12, 29.16, 29.26, 29.31, 29.33, 29.38, 29.55, 29.58, 29.61, 29.65, 126.6, 127.2, 127.5, 127.6, $127.7,127.8,128.0,128.1,128.2,128.7,128.8,129.1,129.2$, 129.4, 130.8, 133.2, 133.6, 134.3, 136.0, 138.6, 140.5. MS m/z (rel. int., \%) $Z$ isomer: $304\left(\mathrm{M}^{+}-\mathrm{C}_{6} \mathrm{H}_{5} \mathrm{Se}, 32.5\right), 136$ (100.0), 91 (29.7); HRMS (ESI): $\mathrm{m} / z$ calcd for $\mathrm{C}_{26} \mathrm{H}_{36} \mathrm{SSe}[\mathrm{M}+\mathrm{H}-\mathrm{S}]^{+}$: 429.2060; found: 429.3037. (Z)- and $(E)-3 e:{ }^{1} \mathrm{H}$ NMR (300 $\left.\mathrm{MHz}, \mathrm{CDCl}_{3}\right) \delta Z$ isomer: 7.54-7.58 (m, 3H); 7.21-7.43 (m, $7 \mathrm{H}) ; 6.82(\mathrm{~s}, 1 \mathrm{H}) ; 2.22$ (t, J 7.5, 2H); 1.45-1.54 (m, 2H); 1.11$1.25(\mathrm{~m}, 4 \mathrm{H}) ; 0.84(\mathrm{t}, J 7.0,3 \mathrm{H}) ; E$ isomer: $7.54-7.58(\mathrm{~m}, 3 \mathrm{H})$; 7.21-7.43 (m, 7H); $6.56(\mathrm{~s}, 1 \mathrm{H}) ; 2.25(\mathrm{t}, J 7.5,2 \mathrm{H}) ; 1.45-1.54$ $(\mathrm{m}, 2 \mathrm{H}) ; 1.11-1.25(\mathrm{~m}, 4 \mathrm{H}) ; 0.84(\mathrm{t}, J 7.0,3 \mathrm{H}) ;{ }^{13} \mathrm{C} \mathrm{NMR}(75$ $\left.\mathrm{MHz}, \mathrm{CDCl}_{3}\right) \delta \mathrm{Z}$ isomer: 14.0, 22.4, 28.1, 31.0, 37.7, 126.7, $128.5,129.0,129.1,129.2,130.2,131.0,132.8,133.8,135.9 ; E$ isomer: 14.0, 22.4, 28.2, 30.9, 37.1, 126.4, 128.8, 129.1, 129.2, 129.7, 130.5, 131.2, 132.9, 134.3, 136.4. MS m/z (rel. int., \%) $Z$ isomer: $362\left(\mathrm{M}^{+}, 46.3\right), 252$ (19.0), 77 (76.0), 43 (100.0); $E$ isomer: $362\left(\mathrm{M}^{+}, 7.55\right), 205$ (7.73), 147 (100.0), 135 (57.5); HRMS (ESI): $m / z$ calcd. for $\mathrm{C}_{19} \mathrm{H}_{22} \mathrm{SSe}[\mathrm{M}+\mathrm{H}]^{+}: 363.0686$; found: 363.0672. (Z)- and (E)-3f: ${ }^{1} \mathrm{H} \mathrm{NMR}\left(300 \mathrm{MHz}, \mathrm{CDCl}_{3}\right)$ $\delta Z$ isomer: 7.21-7.62 (m, 9H), $6.85(\mathrm{t}, J 1.0,1 \mathrm{H}), 2.21(\mathrm{dt}, J$ 1.0 and $7.5,2 \mathrm{H}), 1.49$ (qui, $J 7.5,2 \mathrm{H}), 1.17-1.32(\mathrm{~m}, 4 \mathrm{H}), 0.84$ (t, $J 7.5,3 \mathrm{H}) ; E$ isomer: 7.21-7.62 (m, 9H), $6.50(\mathrm{t}, J 1.0,1 \mathrm{H})$, 2.22 (dt, $J 1.0$ and 7.5, 2H), 1.60 (qui, $J 7.5,2 \mathrm{H}$ ), 1.17-1.32 (m, 4H), $0.85(\mathrm{t}, J 7.5,3 \mathrm{H}) ;{ }^{13} \mathrm{C} \mathrm{NMR}\left(75 \mathrm{MHz}, \mathrm{CDCl}_{3}\right) \delta \mathrm{Z}+E$ isomers: 13.9, 22.3, 22.4, 28.0, 28.1, 30.9, 31.0, 37.0, 37.7, 127.5, 128.5, 129.0, 129.1, 129.2, 129.7, 130.9, 131.2, 131.6, 132.7, 132.8, 135.0, 135.1; MS m/z (rel. int., \%) $Z$ isomer: 396 $\left(\mathrm{M}^{+}, 34.8\right), 253$ (10.5), 77 (51.7), 43 (100.0); $E$ isomer: 396 $\left(\mathrm{M}^{+}, 27.8\right), 209$ (15.0), 147 (44.3), 77 (53.6), 43 (100); HRMS (ESI): $m / z$ calcd. for $\mathrm{C}_{19} \mathrm{H}_{21} \mathrm{ClSSe}[\mathrm{M}+\mathrm{H}-\mathrm{Cl}]^{+}: 360.0615$; found: 360.2335 . (Z)- and (E)-3g: ${ }^{1} \mathrm{H} \mathrm{NMR}\left(300 \mathrm{MHz}, \mathrm{CDCl}_{3}\right.$ ) $\delta Z$ isomer: 7.50-7.57 (m, 2H); 7.25-7.32 (m, 3H); $6.93(\mathrm{t}$, $J 1.0,1 \mathrm{H}) ; 2.28(\mathrm{td}, J 7.5$ and $1.0,2 \mathrm{H}) ; 1.43-1.55(\mathrm{~m}, 4 \mathrm{H})$; $1.17-1.26(\mathrm{~m}, 24 \mathrm{H}) ; 0.83(\mathrm{t}, J 7.0,6 \mathrm{H}) ; E$ isomer: $7.49-7.58$ $(\mathrm{m}, 2 \mathrm{H}) ; 7.23-7.34(\mathrm{~m}, 3 \mathrm{H}) ; 6.04(\mathrm{~s}, 1 \mathrm{H}) ; 2.68$ and $2.72(2 \mathrm{t}$, $J$ 7.5, 2H); 1.43-1.68 (m, 4H); 1.17-1.26 (m, 24H); $0.88(\mathrm{t}$, $J$ 7.0, 3H); 0.83 (t, $J$ 7.0, 3H); $\left.{ }^{13} \mathrm{C} \mathrm{NMR} \mathrm{(75} \mathrm{MHz,} \mathrm{CDCl}_{3}\right) \delta$ $Z+E$ isomer: 14.0, 14.1, 22.3, 22.7, 28.6, 29.2, 29.3, 29.4, 29.5, 29.6, 29.61, 29.62, 29.64, 29.68, 29.7, 30.9, 31.2, 31.9, 39.9, 127.2, 127.3, 127.8, 129.1, 129.2, 129.5, 131.2, 132.6, 132.9, 136.4; MS m/z (rel. int., \%) Z isomer: $454\left(\mathrm{M}^{+}, 21.5\right), 252$ (13.6), 91 (91.4), 55 (100.0); $E$ isomer: $454\left(\mathrm{M}^{+}, 22.5\right), 252$ (15.0), 91 (50.7), 55 (100.0); HRMS (ESI): $m / z$ calcd. for $\mathrm{C}_{25} \mathrm{H}_{42} \mathrm{SSe}$ $[\mathrm{M}+\mathrm{Na}-\mathrm{S}]^{+}:$443.2357; found: 443.3385. (Z)- and $(E)-3 \mathbf{h}:{ }^{1} \mathrm{H}$ NMR (300 MHz, $\left.\mathrm{CDCl}_{3}\right) \delta Z$ isomer: 7.19-7.50 (m, 10H); 6.56 (s, 1H); 3.58 (s, 2H); 2.22 (t, J 7.5, 2H); 1.45-1.58 (m, 2H); 1.17-1.32 (m, 4H); 0.87 (t, J 7.0, 3H); E isomer: 7.19-7.50 (m, $10 \mathrm{H}) ; 6.14(\mathrm{~s}, 1 \mathrm{H}) ; 4.0(\mathrm{~s}, 2 \mathrm{H}) ; 2.42(\mathrm{t}, J 7.5,2 \mathrm{H}) ; 1.45-1.58$ $(\mathrm{m}, 2 \mathrm{H}) ; 1.17-1.32(\mathrm{~m}, 4 \mathrm{H}) ; 0.89(\mathrm{t}, J 7.0,3 \mathrm{H}) ;{ }^{13} \mathrm{C}$ NMR $(75$ $\left.\mathrm{MHz}, \mathrm{CDCl}_{3}\right) \delta \mathrm{Z}+E$ isomers: 14.0, 22.4, 28.0, 28.4, 31.1, 31.2, $35.4,36.5,37.9,43.2,125.7,126.4,127.0,127.2,127.3,128.3$, $128.4,128.5,128.7,128.8,129.1,129.3,130.6,131.3,132.5$, 132.8, 136.7, 137.3, 137.7; MS m/z (rel. int., \%) $Z$ isomer: 376 (M+, 9.6), 205 (8.0), 147 (18.6), 91 (100.0); $E$ isomer: $376\left(\mathrm{M}^{+}\right.$, 7.6), 204 (6.8), 147 (16.7), 91 (100); HRMS (ESI): m/z calcd. for $\mathrm{C}_{20} \mathrm{H}_{24} \mathrm{SSe}[\mathrm{M}+\mathrm{K}]^{+}$: 413.0409; found: 413.2725.

16. Wang, S.-X.; Li, J.-T.; Yang, W.-Z.; Li, T.-S.; Ultrason. Sonochem. 2002, 9, 159.

Submitted: May 5, 2010 Published online: August 5, 2010 